ITP-Budapest 562

\title{
Geometry of $W$-algebras from the affine Lie algebra point of view
}

\author{
Z. Bajnok ${ }^{1}$ and D. Nógrádi ${ }^{1,2}$ \\ December, 2000 \\ ${ }^{1}$ Institute for Theoretical Physics, Eötvös University \\ Budapest 1117, Pázmány Péter sétány 1/A, Hungary \\ ${ }^{2}$ Institute Lorentz, University of Leiden \\ P.O. Box 9506, NL-2300 RA Leiden, The Netherlands
}

\begin{abstract}
To classify the classical field theories with $W$-symmetry one has to classify the symplectic leaves of the corresponding $W$-algebra, which are the intersection of the defining constraint and the coadjoint orbit of the affine Lie algebra if the $W$-algebra in question is obtained by reducing a WZNW model. The fields that survive the reduction will obey non-linear Poisson bracket (or commutator) relations in general. For example the Toda models are well-known theories which possess such a non-linear $W$ symmetry and many features of these models can only be understood if one investigates the reduction procedure. In this paper we analyze the $S L(n, \mathbb{R})$ case from which the so-called $W_{n}$-algebras can be obtained. One advantage of the reduction viewpoint is that it gives a constructive way to classify the symplectic leaves of the $W$-algebra which we had done in the $n=2$ case which will correspond to the coadjoint orbits of the Virasoro algebra and for $n=3$ which case gives rise to the Zamolodchikov algebra. Our method in principle is capable of constructing explicit representatives on each leaf. Another attractive feature of this approach is the fact that the global nature of the $W$-transformations can be explicitly described. The reduction method also enables one to determine the classical highest weight (h. w.) states which are the stable minima of the energy on a $W$-leaf. These are important as only to those leaves can a highest weight representation space of the $W$-algebra be associated which contains a classical h. w. state.
\end{abstract}

\section{Introduction}

$W$-algebras have attracted a great interest since their first appearance 11]. They are extensions of the Virasoro algebra by higher spin currents in general in such a way that the Poisson bracket or commutator of the currents give non-linear terms

$$
\left[W_{i}, W_{j}\right]=P_{i j}(W)
$$

where $P_{i j}(W)$ is a polinomial of the currents. Such extensions are relevant not only in the classification of two dimensional conformal field theories but also in describing various statistical physical models.

There are various ways to obtain the so-called $W_{n}$-algebras on both quantum and classical level; see [25, 26] for the quantum field theoretical aspects where the degenerate representations, the corresponding null vectors and quantum highest weights were constructed for $W_{3}$ and was already pointed out how this algebra was related to $s l(3)$. Later it was shown in [1] that the Toda models which carry the $W_{n}$-algebras as symmetry algebras are Hamiltonian reductions of the Wess-Zumino-Novikov-Witten (WZNW) models. The classical interpretation of the $W_{n}$-algebras was discussed from a similar viewpoint also in [30] where the conformal Ward-identities were also derived. In [27, 28] a different realization was proposed, the classical $W_{n}$-algebras were related to certain embeddings of two dimensional manifolds into $2 n$ dimensional Kähler manifolds which construction makes global considerations possible and quite explicit. A link between the quantum and classical levels was established in [29] and [2]. The classical realization was studied geometrically in [29] where the equation of motion was originated from immersions of affine surfaces of constant mean curvature in affine space and was shown to be the classical $(c \rightarrow-\infty)$ limit of Zamolodchikov's null vector equation. In [2] the null vector equation was obtained as quantization of the classical equation of motion for $W_{3}$. 
Once the classical $W$-algebra is given - no matter where it originates from, we have seen there are various ways to arrive at them - one can ask about its symplectic leaves and classical highest weight states. This is the subject of the present paper. We will follow the gauged WZNW model construction as this approach is the most useful one for our purposes as it will become clear, but we would like to stress that other classical interpretations of $W$-algebras mentioned above are equally good and the problems we address are independent of how the classical algebras were arrived at.

A "phase space" associated to a Poisson manifold is one of its symplectic leaves. For the exploration of the leaves we will use the well-known classical result stating roughly that if second class constraints are imposed on a Poisson manifold $P$ resulting in a submanifold $C \subset P$ (which is also Poisson in a canonical way) then the symplectic leaves $M \subset C$ of $C$ coincide with the symplectic leaves $S \subset P$ of $P$ intersected by $C$.

In our case the Poisson manifold $P$, as we have Kac-Moody symmetry, is the Kac-Moody algebra with its canonical Poisson structure that comes from the Lie algebra structure. Its symplectic leaves $S$ are the connected components of its coadjoint orbits and the $W$-algebra lives on the constraint submanifold $C$ determined by the Toda-type constraints. The statement above means that the symplectic leaves of the $W$-algebra are nothing else then the connected components of the intersection of the constraint and the Kac-Moody coadjoint orbits. This implies that to classify the classical theories with $W$-symmetry, which is the same as to classify the symplectic leaves of the $W$-algebra, one has to classify the connected components of the intersection of the Kac-Moody coadjoint orbits and the constraint hypersurface.

Exploring the symplectic leaves in this way enables one in principle to give explicit representatives on each $W$-leaf which was carried out in the $n=2$ case but became technically quite cumbersome already if $n=3$

The issue of the classical h. w. state i.e. a stable minimum of the energy can also be handled by the reduction as the energy functional and its first and second variation on a $W$-leaf will first be considered on the Kac-Moody coadjoint orbit and then restricted to the intersection. We will determine which orbits contain a classical h. w. state which will indicate that these can give rise to a highest weight representation space of the $W$-algebra.

Yet another advantage of the reduction viewpoint is that we can implement the $W$-transformations as gauge preserving Kac-Moody transformations. These were given in [1] on the Lie algebra level but our analysis makes it possible to explicitly describe them on the group level. These are important for understanding the global nature of $W$-transformations.

Our motivation for analyzing the classical geometry of $W$-algebras is at least twofold. First consider quantization. The quantization of $W$-algebras started by a free field construction [14, then a BRST method [16] was adopted to produce their quantum counterparts. None of the approaches above however, relied on the geometry of $W$-algebras loosing useful information in this way. For example one possible quantization procedure would be the geometric quantization of the symplectic leaves for which it is essential to explore the underlying geometry.

Our second motivation comes from a more mathematical context. There is a completely different viewpoint of the classification problem which has its origin in the field of integrable models. In this approach the classification of symplectic leaves is related to the homotopy classes of certain non-degenerate curves on projective space or sphere which problem is still unsolved in the general case [6, 9]. We will try to make the relation of the two approaches clear but will not elaborate much on this direction. Note that this formulation is close in spirit to a third approach which addresses the problem throught the second Gel'fand-Dikii bracket [12].

In section 2 we will summarize the well-known setting of the WZNW models concentrating on the symmetry properties. Section 3 will be a summary of those features of the geometry of loop groups and their central extensions which are necessary for our considerations. This section will closely follow [33]. After these rather general remarks we will set the stage for the reduction procedure in the case of the $S L(n, \mathbb{R})$ WZNW model in section 1 for which the main reference is [1]. The detailed analysis will be done in the two simplest cases; the $S L(2, \mathbb{R})$ case will amount to the classification of the coadjoint orbits of the Virasoro algebra and the well-known result will be recovered $[6,17,18,19]$. The $S L(3, \mathbb{R})$ case in section 6 will produce the simplest $W$-algebra, the so-called Zamolodchikov algebra and we will mainly focus on the behaviour of the energy functional on the $W$-leaves rather then giving explicit representatives on each of them. We will summerize our and comment on other results and open questions in section 7 . 


\section{WZNW models}

The usual action of the WZNW model on a connected, non-compact, simple Lie group is the following:

$$
S=\frac{k}{8 \pi} \int_{\Sigma} d^{2} x \eta^{\mu \nu} \operatorname{Tr}\left(g^{-1} \partial_{\mu} g\right)\left(g^{-1} \partial_{\nu} g\right)-\frac{k}{12 \pi} \int_{B} \operatorname{Tr}\left(g^{-1} d g\right)^{3},
$$

where $k$ is the coupling constant, $\Sigma=S^{1} \times \mathbb{R}$ is the $1+1$ dimensional Minkowski space, $B$ is a 3-manifold whose boundary is $\Sigma, \eta^{\mu \nu}$ is the Lorentzian metric on $\Sigma$, and $g: \Sigma \longrightarrow G$ is the dynamical field. If a certain element in $H^{3}(G)$ is not zero then $k$ has to be an integer otherwise the action or rather the transition amplitudes are ill defined [31].

The equations of motion look in terms of the currents in light-cone coordinates as

$$
\partial_{-} J_{+}=0, \quad \partial_{+} J_{-}=0,
$$

where $J_{+}, J_{-}: \Sigma \longrightarrow \mathfrak{g}$ are the Lie algebra valued currents,

$$
J_{+}=k \partial_{+} g g^{-1}, \quad J_{-}=-k g^{-1} \partial_{-} g,
$$

and $x_{ \pm}=t \pm x$ are the light-cone coordinates on $\Sigma$. The solutions are simply that $J_{+}$depends on $x_{+}, J_{-}$ on $x_{-}$only, which means they are periodic functions of one variable. This implies that the solution in the language of the $g$ field is

$$
g\left(x_{+}, x_{-}\right)=g_{+}\left(x_{+}\right) g_{-}\left(x_{-}\right)
$$

where $g_{+}$and $g_{-}$are quasiperiodic group valued fields which means $g_{+}\left(x_{+}+2 \pi\right)=g_{+}\left(x_{+}\right) M$ and that $g_{-}\left(x_{-}-2 \pi\right)=M^{-1} g_{-}\left(x_{-}\right)$for some $M \in G$ monodromy.

The action of an $x_{+}$dependent chiral $h\left(x_{+}\right)$Kac-Moody transformation on the fields are:

$$
\begin{aligned}
g & \longmapsto h g h(0)^{-1} \\
J_{+} & \longmapsto h J_{+} h^{-1}+k \partial_{+} h h^{-1},
\end{aligned}
$$

and analogous formulas hold for $J_{-}$. These chiral transformations are symmetries of the action and we will see that these are nothing but the coadjoint action of the centrally extended loop group, and to make the identification more transparent $h(0)^{-1}$ is defined into the transformation of $g$. This modification is actually a combination of the left local, $g\left(x_{+}, x_{-}\right) \longmapsto h\left(x_{+}\right) g\left(x_{+}, x_{-}\right)$, and the right global, $g\left(x_{+}, x_{-}\right) \longmapsto$ $g\left(x_{+}, x_{-}\right) h(0)^{-1}$, transformations which are both symmetries of the action. The conservation laws that correspond to these symmetries coincide with the equations of motion.

From this point forward we will only deal with the "+" chirality and $J, g$ and $x$ will mean $J_{+}, g_{+}$and $x_{+}$. So $g$ and $J$ are functions of one variable, $J$ is periodic $g$ is quasiperiodic only.

As the $J$ current generates the (11) transformations which will turn out to be the coadjoint action of the centrally extended loop group the Poisson brackets of its components coincide of course with the commutation relations of the Kac-Moody algebra. Let $\left\{t^{a}\right\}$ be a basis of $\mathfrak{g}$, then $J(x)=J_{a}(x) t^{a}$ and

$$
\left\{J_{a}(x), J_{b}(y)\right\}=f_{a b}^{c} J_{c}(x) \delta(x-y)+k K_{a b} \delta^{\prime}(x-y),
$$

where $\delta(x)$ is the $2 \pi$ periodic $\delta$-function, $f_{a b}^{c}$ are the Lie algebra structural constants and $K_{a b}$ is the Killing form.

\section{Loop groups and their central extensions and coadjoint orbits}

Let $G$ be a connected and simple Lie group. The loop group $L G$, associated to $G$ is the group of smooth $S^{1} \longrightarrow G$ mappings with pointwise multiplication [33]. The following observation will be important: if $G$ is not simply connected then $L G$ is not connected as the connected components of $L G$ are labeled by the fundamental group of $G$.

Let us parametrize the circle with $x$ ranging from 0 to $2 \pi$. The map $h \longmapsto\left(h(0), h h(0)^{-1}\right)$ is a diffeomorphism between $L G$ and $G \times \Omega G$ where $\Omega G$ is the group of such loops that start at the identity. On $G \times \Omega G$ the group multiplication is a semi-direct product:

$$
(A, \gamma)(B, \eta)=\left(A B, \gamma A \eta A^{-1}\right), \quad A, B \in G, \quad \gamma, \eta \in \Omega G,
$$

so $\gamma(0)=\eta(0)=1$. The Lie algebra of $L G, L \mathfrak{g}$, is the space of smooth $S^{1} \longrightarrow \mathfrak{g}$ mappings, the Lie algebra of $\Omega G, \Omega \mathfrak{g}$, is the space of such loops that start at the origin. 
The Kac-Moody algebras are the central extensions of $L \mathfrak{g}$, they are denoted by $\hat{\mathfrak{g}}$. Since $G$ is simple the extension by $\mathbb{R}$ is unique up to a scalar factor and the corresponding cocycle is $c\left(J_{1}, J_{2}\right)=\left\langle J_{1}^{\prime}, J_{2}\right\rangle$, where

$$
\left\langle J_{1}, J_{2}\right\rangle=\int_{0}^{2 \pi} \operatorname{Tr} J_{1}(x) J_{2}(x) \frac{d x}{2 \pi}
$$

is a non-degenerate form on $L \mathfrak{g}$. Note that the cocycle $c: L \mathfrak{g} \times L \mathfrak{g} \longrightarrow \mathbb{R}$ is degenerate, its kernel consists of precisely the constant elements but if it is restricted to $\Omega \mathfrak{g} \times \Omega \mathfrak{g}$ it will turn non-degenerate as the constant elements in $\Omega \mathfrak{g}$ are zero.

While considering the coadjoint orbits of the centrally extended loop groups it is clearly enough to deal with the action of $L G$ and $L \mathfrak{g}$ on $\hat{\mathfrak{g}}$ as we have a central extension at hand. Note that we have not touched the delicate issue of central extensions on the group level and we will continue to ignore this subtlety. It is easy to check that the adjoint action of $K \in L \mathfrak{g}$ and $h \in L G$ on $\hat{\mathfrak{g}}$ is

$$
\begin{gathered}
K \cdot(J, k)=([K, J], c(K, J)), \\
h \cdot(J, k)=\left(h J h^{-1}, k-\left\langle h^{-1} h^{\prime}, J\right\rangle\right) .
\end{gathered}
$$

The coadjoint action will be considered also on $\hat{\mathfrak{g}}$ after identifying the dual of $L \mathfrak{g}$ with $L \mathfrak{g}$ by the (2) scalar product and turns out to be

$$
h \cdot(J, k)=\left(h J h^{-1}+k h^{\prime} h^{-1}, k\right) \text {, }
$$

where $\cdot$ now of course means the coadjoint action. In the first component we have the symmetry transformation (1) of the WZNW model and the central term is invariant, so we will think of the coadjoint action of the centrally extended loop group as an action of $L G$ on $L \mathfrak{g}$ with a given $k$ fixed. The corresponding orbit will be referred to as the Kac-Moody coadjoint orbit or simply as the coadjoint orbit.

Let us consider the quasiperiodic group valued field of the WZNW model with $M \in G$ monodromy for analyzing the coadjoint orbits. The association of $J$ to $g$ via the $J=k g^{\prime} g^{-1}$ definition is one-to-one between the space of quasiperiodic group valued fields and $L \mathfrak{g}$ if we add the $g(0)=1$ initial condition which will mean that $M=g(2 \pi)$. Note that this initial condition is the reason for including the constant term in (1). To a given $J(x)$ corresponds the path ordered $g(x)=\mathrm{P} \exp \left(\int_{0}^{x} J(y) d y\right)$. If the transformation of $J$ is $J \longmapsto h J h^{-1}+k h^{\prime} h^{-1}$ then the associated $g$ transforms as $g \longmapsto h g h(0)^{-1}$ which are the symmetry transformations of the WZNW model. The monodromy also changes to $M \longmapsto h(0) M h(0)^{-1}$. This means that if two $J_{\mathrm{S}}$ are on the same orbit then the corresponding monodromies are conjugated in $G$. The other way around take two elements in $L \mathfrak{g}, J_{1}$ and $J_{2}$ and let the corresponding quasiperiodic fields and monodromies be $g_{1}, g_{2}$ and $M_{1}$ and $M_{2}$. Let us assume that the monodromies are conjugated by an $A \in G, M_{2}=A M_{1} A^{-1}$, then $g_{2} A g_{1}^{-1}$ is periodic and takes $J_{1}$ to $J_{2}$ which means they are on the same orbit.

To summarize the above considerations there is an equivariant correspondence between the conjugacy classes of $G$ and the Kac-Moody coadjoint orbits which fact greatly simplifies the study of the latter. For example it is fairly straightforward to determine the isotropy subgroup, $H_{J} \subset L G$, of a given $J$ because if $h$ leaves $J$ fixed then it must be true that $g=h g h(0)^{-1}$ and $h(0)$ commutes with the corresponding monodromy, which means that $h$ is such that $h=g A g^{-1}$ where $h(0)=A$ is any element which commutes with $M$. This implies that the map $h \longmapsto h(0)$ is an isomorphism from $H_{J}$ to the commutant subgroup of $M$ in $G$, hence we will think of $H_{J}$ as a subgroup of $G$.

In general any orbit can be realized as a homogenous manifold which gives in our case the realization $\mathcal{O}_{J}=L G / H_{J}=(G \times \Omega G) / H_{J}$ where $\mathcal{O}_{J}$ is the coadjoint orbit of $J$. As $H_{J}$ can be thought of as a subgroup of $G$ one might suspect that the factorization only takes place in the first $(G)$ component. To make this idea precise consider the map:

$$
\begin{aligned}
\pi: \mathcal{O}_{J} & \longrightarrow G / H_{J} \\
h \cdot J & \longmapsto[h(0)],
\end{aligned}
$$

where - is the coadjoint action and $[h(0)]$ is the class of $h(0)$ in $G / H_{J}$. This map is easily seen to be well defined. It is clear that $\pi^{-1}([A])$ is nothing other then those elements in $\mathcal{O}_{J}$ whose monodromies are $A M A^{-1}$ and that on this set $\Omega G$ acts freely as if the monodromy of $g$ is $A M A^{-1}$ and $\gamma \in \Omega G$ then the monodromy of $\gamma g \gamma(0)^{-1}=\gamma g$ is the same. Local trivializability can also be established. Summarizing, the coadjoint orbits of Kac-Moody groups are principal $\Omega G$-bundles over $G / H_{J}$, hence all the infinite dimensional subtleties were isolated in the fiber and is the same for all orbit and the orbit specific features were captured in the finite dimensional homogenous manifold $G / H_{J}$. 
Let us elaborate a little more on the important special case when the monodromy $M$ has a logarithm in $\mathfrak{g}$ i.e. it is of the form $M=\exp (X)$ for some $X \in \mathfrak{g}$. Having such an $M$ at hand an obvious choice for the quasiperiodic field with this monodromy is $g(x)=M^{\frac{x}{2 \pi}}$. To this $g$ corresponds the constant current $J=\frac{1}{2 \pi} X$ which shows that in this special case the orbit contains a constant representative which is not true in general for non-compact groups. Put it the other way it is quite obvious that if an orbit contains a constant representative then the corresponding monodromy has a logarithm.

The isotropy subgroup of a constant $J=\frac{1}{2 \pi} X$ consists of only those constant elements in $L G$ which commute with $M$ which is the same as the isotropy subgroup of $X, H_{X}$, with respect to the adjoint action of $G$ on $\mathfrak{g}$. Recall that the (co)adjoint orbit of $X, \mathcal{O}_{X}$, can be identified with $G / H_{X}$ which makes it clear that in this case the $\mathcal{O}_{J}$ orbit is a principal $\Omega G$-bundle over $\mathcal{O}_{X}$ :

$$
\begin{aligned}
\mathcal{O}_{J} & \longrightarrow \mathcal{O}_{X} \\
h \cdot J & \longmapsto h(0) X h(0)^{-1}
\end{aligned}
$$

and the map

$$
\begin{aligned}
\mathcal{O}_{J} & \longrightarrow \mathcal{O}_{X} \times \Omega G \\
h \cdot J & \longmapsto\left(h(0) X h(0)^{-1}, h h(0)^{-1}\right)
\end{aligned}
$$

trivializes $\mathcal{O}_{J}$. Note that the (3) bundle can be trivialized in any case over $G / H_{J}$ not only when $M$ possesses a logarithm but in general the trivializing map is more complicated.

The chiral energy in the WZNW model is of the usual form

$$
E(J)=\frac{1}{2}\langle J, J\rangle=\frac{1}{2} \int_{0}^{2 \pi} \operatorname{Tr} J^{2}(x) \frac{d x}{2 \pi}
$$

whose critical points on the orbit are the constant elements because if $\delta J=[K, J]+k K^{\prime}$ for a $K \in L \mathfrak{g}$ then

$$
\delta E=k \int_{0}^{2 \pi} \operatorname{Tr} J(x) K^{\prime}(x) \frac{d x}{2 \pi}
$$

which is obviously zero only for any $K$ if $J$ is constant. This can also be seen by noting that $E(J)$ generates the rigid rotations of the circle and the critical points should be invariant, hence they must be constant. The critical points are minima of the energy only if the second variation is positive semi-definite, which turns out to be at a critical point evaluated on two elements $K_{1}, K_{2} \in L \mathfrak{g}$

$$
\delta_{2} \delta_{1} E=k \int_{0}^{2 \pi} \operatorname{Tr}\left(J\left[K_{1}^{\prime}(x), K_{2}(x)\right]+k K_{1}^{\prime}(x) K_{2}^{\prime}(x)\right) \frac{d x}{2 \pi} .
$$

which is symmetric in $K_{1}$ and $K_{2}$ for a constant $J$ as it should be. The positive semi-definiteness of this quadratic form is equivalent to the positive semi-definiteness of the operator

$$
-k^{2}\left(\frac{d}{d x}\right)^{2}+k \operatorname{ad} J \frac{d}{d x}
$$

on $\mathrm{Lg}$.

\section{$4 \quad S L(n, \mathbb{R})$ WZNW model and reduction}

Systems with $W$-symmetry can be obtained from WZNW models based on maximally non-compact Lie groups by imposing appropriate constraints. In general an $s l(2, \mathbb{R})$ embedding is needed in the Lie algebra in order to define the constraints but we will only deal with the principal embedding in the $S L(n, \mathbb{R})$ case from which generalization to other groups and embeddings is straightforward. From now on $J / k$ is denoted by $J$ (assuming that $k \neq 0$ ) and $k$ will not appear explicitly in the formulas. The constraint is the fixing of certain components of the current:

$$
J_{\alpha}(x)=\left\{\begin{array}{lll}
1 & \text { if } & \alpha \in \Delta_{-} \\
0 & \text { if } & \alpha \in \Phi_{-} \backslash \Delta_{-}
\end{array}\right.
$$


if the Cartan basis is chosen in $\mathfrak{g}$ and where $\Phi_{-}$is the set of negative roots and $\Delta_{-}$is the set of simple negative roots.

These constraints mean that the form of $J$ is restricted in the following way:

$$
J=\left(\begin{array}{ccccccc}
* & * & . & . & . & \cdot & * \\
1 & * & * & & & & \cdot \\
0 & 1 & * & * & & & \cdot \\
\cdot & 0 & 1 & & & & \cdot \\
\cdot & & & . & & & \cdot \\
. & & & & . & * & \cdot \\
0 & . & . & . & 0 & 1 & *
\end{array}\right) .
$$

It is easy to show that these are first class constraints in the sense of Dirac and generate gauge transformations which are precisely the transformations generated by the components of the current corresponding to the positive roots. One possible gauge fixing is the Wronsky gauge in which the current takes the form

$$
J=\left(\begin{array}{ccccccc}
0 & W_{2} & W_{3} & W_{4} & . & . & W_{n} \\
1 & 0 & 0 & . & . & . & 0 \\
0 & 1 & 0 & & & & . \\
. & 0 & 1 & & & & . \\
. & & & . & & & . \\
. & & & & . & 0 & . \\
0 & . & . & . & 0 & 1 & 0
\end{array}\right) .
$$

One can think of the above form as the constrained form of the current by second class constraints and can calculate the Poisson bracket of the reduced $W_{2}, \ldots, W_{n}$ fields three different ways. As gauge invariant quantities one can use the original Poisson bracket, or as fields left from second class constraints one can adopt the Dirac formalism. The third possibility is based on the fact (more or less definition) that the Poisson bracket taken with a $W_{m}$ field is the same as the infinitezimal $W$-transformation generated by it and the brackets can be read off from the transformation properties. The bracket of the $W_{2}=L$ energy-momentum tensor with itself gives the Virasoro algebra of course and suitable combinations of the other $W_{m}$ fields transform as primary fields of weight $m$ under conformal transformations. The bracket of two $W_{m}$ fields $(m \neq 2)$ gives non-linear expressions in the others. This Poisson algebra is called the $W_{n}$-algebra.

The energy-momentum tensor generates the conform transformations out of which the rigid rotations are generated by the chiral energy $E=L_{0}=\int_{0}^{2 \pi} L(x) \frac{d x}{2 \pi}$, hence the critical points of the energy on a $W$-leaf are those points which are invariant under rigid rotations i.e. all $W_{m}$ fields must be constant which means that this property has survived the reduction.

The constraint on the current gives also restrictions on the $g$ field. For the current to have the gauge fixed (7) form $g$ must take the form

$$
g=\left(\frac{\frac{\psi^{(n-1)}}{\vdots}}{\frac{\psi^{\prime}}{\psi}}\right)
$$

where $\boldsymbol{\psi}: \mathbb{R} \longrightarrow \mathbb{R}^{n}$ is a quasiperiodic row vector with $M$ monodromy and $\boldsymbol{\psi}^{(n-1)}$ is the $(n-1)^{t h}$ derivative. The $\operatorname{det} g=1$ and $g(0)=1$ conditions mean further restrictions on $\boldsymbol{\psi}$ and it follows from the $J=g^{\prime} g^{-1}$ relation that its components are the linearly independent solutions of the

$$
\psi^{(n)}=\sum_{m=2}^{n-1} W_{m} \psi^{(n-m)}
$$

$n^{\text {th }}$ order linear differential equation which is the field equation of the reduced theory in one chirality. Note that in case $n=2$ equation (9) turns into the Hill equation which was used in [17 for analyzing the coadjoint orbits of the Virasoro algebra. Also note that if $\psi$ in $(8)$ is multiplied by any periodic 
function $r(x)$ then det $g$ picks up a factor of $r^{n}(x)$, which shows that the length of $\boldsymbol{\psi}$ is irrelevant, it can be thought of as a quasiperiodic curve in $\mathbb{R} P^{n-1}$ or $S^{n-1}$ and the $\operatorname{det} g \neq 0$ condition is a certain non-degeneracy condition which makes it clear that the symplectic leaf classification problem is equivalent to the determination of homotopy types of quasiperiodic non-degenerate curves on projective space or the sphere. This approach is followed in $[6,9]$.

The Kac-Moody transformations which preserve the gauge fixed form of the current or equivalently the (8) constrained form of $g$ are also symmetries of the reduced model, these are the $W$-transformations. To give an explicit description of them let us consider the constrained $g$ field in the form of (8). It is not difficult to show that this form is preserved by an $h \in L G$ Kac-Moody transformation

$$
h=\left(\begin{array}{c}
\frac{\mathbf{a}_{n}}{\vdots} \\
\frac{\mathbf{a}_{2}}{\mathbf{a}_{1}}
\end{array}\right),
$$

only if the $\mathbf{a}_{m}: S^{1} \longrightarrow \mathbb{R}^{n}$ row vectors satisfy the $\mathbf{a}_{m+1}=\mathbf{a}_{m}^{\prime}+\mathbf{a}_{m} J, m=1 \ldots n-1$ recursion relation whose solution is

$$
\mathbf{a}_{m+1}(x)=\mathbf{a}_{1}(x)\left(\frac{d}{d x}+J(x)\right)^{m},
$$

where differentiation acts on the left. If the current is not in the gauge fixed form then the expression (10) gives those transformations which bring it to the gauge. As a consequence of the recursion relation any component of $h$ can be expressed in terms of $\mathbf{a}_{1}$, the only restriction on it is the det $h=1$ condition which makes it possible to express the last component of $\mathbf{a}_{1}$ with the others on the infinitezimal (Lie algebra) level. The next to the last component generates the conformal the others the $W$-transformations.

Based on the above reduction procedure it is not hard to find the number of independent vectorfields that leave all the $W_{m}$ fields fixed by the corresponding $W$-transformation. Because if it leaves them fixed one can neglect the constraint hypersurface and the answer is the dimension of the isotropy subgroup $H_{J} \subset G$ of $J$. Attacking this problem by brut-force methods would involve the determination of the number of independent solutions of complicated differential equations subject to periodic boundary conditions which is in general quite cumbersome. This direct method was used in 118 for determining the possible dimensions in the case of the Virasoro algebra.

To list all classical models with such a $W$-symmetry one has to classify the symplectic leaves of the non-linear Poisson structure of the $W_{m}$ fields. These can be obtained, just as in the finite dimensional cases [21, 22], as the intersection of the coadjoint orbit of the original symmetry algebra, which is of course the $S L(n, \mathbb{R})$ Kac-Moody algebra, and the constraint hypersurface as it can be implemented by second class constraints. Or more precisely as a symplectic leaf is connected by definition, the connected components of the intersection should be determined.

\section{$5 \quad S L(2, \mathbb{R})$ Kac-Moody and Virasoro algebra}

The only component of the current that survives the reduction is the $W_{2}=L$ energy-momentum tensor which means that applying our method in this case will result in the classification of the coadjoint orbits of the Virasoro algebra and should reproduce the well-known results [6, 17, 18, 19]. In [17] the authors classified the Virasoro orbits through the study of the Liouville (or Hill) equation, hence our method will be a constructive way of producing the conformally inequivalent solutions of the Liouville (or Hill) equation.

Also important to note that since $\pi_{1}(S L(2, \mathbb{R}))=\mathbb{Z}$, which is given by the winding number of the second row of the $S L(2, \mathbb{R})$ matrix, every Kac-Moody orbit consists of $\mathbb{Z}$ connected components. If a $J$ current is given on a specific component of an orbit then to take it to other components of the same orbit one can apply for example the

$$
T_{n}(x)=\left(\begin{array}{cc}
\cos (n x) & -\sin (n x) \\
\sin (n x) & \cos (n x)
\end{array}\right)
$$

Kac-Moody transformation which has winding number $n$, where $n$ is any integer. As the symplectic leaves are connected one has to determine the connected components of the intersection of the Kac-Moody orbit and the constraint hypersurface. It will turn out that the constraint either intersects each component of 
the Kac-Moody orbit in one piece or it does not intersect it at all. This means that the Virasoro orbits can be labelled by the conjugacy class of the monodromy matrix and by those $n$ s to which component belongs an intersection. The issue of the existence of the intersection will be dealt with in the following way, if a current is given on a specific component of a Kac-Moody orbit we will try to transform it to the desired $\left(\begin{array}{cc}0 & L \\ 1 & 0\end{array}\right)$ form by a Kac-Moody transformation that has winding number zero in order to stay on the same component. If this can be done the resulting $L$ will immediately be a representative of the Virasoro orbit, if it can not be done then the component in question has no intersection with the constraint hypersurface.

Before the details note one more qualitative remark: according to section the energy-momentum tensor can be left fixed by 1 or 3 generators only as the group $S L(2, \mathbb{R})$ has only 1 and 3 dimensional isotropy subgroups, so in the realization $\operatorname{Diff}\left(S^{1}\right) / H$ of the Virasoro orbits the dimension of $H$ can only be 1 or 3 as it is well-known. In the detailed analysis we will give the vectorfields for each representative of the Virasoro orbit which leaves it fixed under the coadjoint Virasoro action.

After imposing the constraint and the $\operatorname{det} g=1$ condition only one free function $\phi$ remains in $g$ :

$$
g=\left(\begin{array}{cc}
\left(\frac{\sin \phi}{\sqrt{\phi^{\prime}}}\right)^{\prime} & \left(\frac{\cos \phi}{\sqrt{\phi^{\prime}}}\right)^{\prime} \\
\frac{\sin \phi}{\sqrt{\phi^{\prime}}} & \frac{\cos \phi}{\sqrt{\phi^{\prime}}}
\end{array}\right),
$$

where of course $\phi^{\prime}>0$. The $g(0)=1$ initial condition and quasiperiodicity of $g$ implies that

$$
\begin{aligned}
\phi(0) & =0, & e^{i \phi(2 \pi)} & =\frac{d+i c}{\sqrt{d^{2}+c^{2}}} \\
\phi^{\prime}(0) & =1, & \phi^{\prime}(2 \pi) & =\frac{1}{d^{2}+c^{2}} \\
\phi^{\prime \prime}(0) & =0, & \phi^{\prime \prime}(2 \pi) & =-2 \frac{c d+a b}{\left(d^{2}+c^{2}\right)^{2}},
\end{aligned}
$$

where $M=\left(\begin{array}{ll}a & b \\ c & d\end{array}\right)$ is the monodromy matrix. The condition on $\phi(2 \pi)$ and the fact that $\phi$ is monotonically increasing implies that one can find an integer $N \geq 0$ such that

$$
\phi(2 \pi)=\alpha+2 \pi N,
$$

where $e^{i \alpha}=\frac{d+i c}{\sqrt{d^{2}+c^{2}}}$ and $0<\alpha \leq 2 \pi$. It does not make sense to talk about the winding number of $\phi$ as it is only quasiperiodic but the $N \geq 0$ integer just defined describes its winding in a certain sense. More precisely let $J_{1}$ and $J_{2}$ be two gauge fixed elements on the same Kac-Moody orbit but not necessarily on the same component. Their monodromies are conjugated by an $A \in S L(2, \mathbb{R}), M_{2}=A M_{1} A^{-1}$. Let $g_{1}$ and $g_{2}$ be the quasiperiodic fields associated to them and since they are gauge fixed they can be expressed with a $\phi_{1}$ and $\phi_{2}$ functions whose invariant are denoted by $N_{1}$ and $N_{2}$. Then $J_{1}$ and $J_{2}$ are on the same component if and only if $N_{1}=N_{2}$. One has only to prove that the winding number of $h=g_{2} A g_{1}^{-1}: S^{1} \longrightarrow S L(2, \mathbb{R})$ which takes $J_{1}$ to $J_{2}$ is zero if and only if $N_{1}=N_{2}$ but this is not difficult. So the $N$ invariant describes on which Kac-Moody component is the $J$ relative to a prescribed representative.

The space of $\phi$ functions with fixed monodromy and $N$ invariant, subject to the (11) conditions is clearly connected so if the monodromy is fixed the constraint hypersurface can intersect each component in maximum one piece. Besides, if the monodromy is changed within its conjugacy class then in those components where there was an intersection it will continue to be so, which means that the constraint hypersurface intersects a Kac-Moody component in either one piece or not at all. It also turns out from this analysis that since $N \geq 0$ only those Kac-Moody components can have an intersection with the constraint that are "above" the prescribed representative which fact will be transparent in each specific case in the detailed analysis.

In each gauge fixed current the total degrees of freedom is in the corresponding $\phi$. The energymomentum tensor can be expressed as:

$$
L=-\phi^{\prime 2}+\frac{1}{2} S(\phi)
$$

where $S(\phi)=\frac{3}{2} \frac{\phi^{\prime \prime 2}}{\phi^{\prime 2}}-\frac{\phi^{\prime \prime \prime}}{\phi^{\prime}}$ is the Schwarzian derivative. We have brought any $L$ to the above form which is quite remarkable as if it would be true that $\phi(x+2 \pi)=\phi(x)+2 \pi$ then this formula would mean that 
any $L$ is on the orbit of -1 which is of course not true because the complicated (111) formulas hold for $\phi(x+2 \pi)$ instead of the previous one.

To classify the Kac-Moody and Virasoro orbits one has to present a complete list of conjugacy classes. There are four distinct types of these depending on the isotropy subgroup $H$.

- Elliptic classes, those monodromies belong here for which $|\operatorname{Tr} M|<2$,

$$
H=\left\{\left(\begin{array}{cc}
\cos (t) & -\sin (t) \\
\sin (t) & \cos (t)
\end{array}\right) \mid t \in(0,2 \pi]\right\},
$$

representatives:

$$
M=\left(\begin{array}{cc}
\cos (2 \pi \omega) & -\sin (2 \pi \omega) \\
\sin (2 \pi \omega) & \cos (2 \pi \omega)
\end{array}\right), \omega \in(0,1), \omega \neq \frac{1}{2} .
$$

- Hyperbolic classes, those monodromies belong here for which $|\operatorname{Tr} M|>2$,

$$
H=\left\{\left(\begin{array}{cc}
t & 0 \\
0 & t^{-1}
\end{array}\right) \mid t \in \mathbb{R}^{*}\right\}
$$

representatives:

$$
M= \pm\left(\begin{array}{cc}
e^{2 \pi b} & 0 \\
0 & e^{-2 \pi b}
\end{array}\right), b>0 .
$$

- Parabolic classes, those monodromies belong here for which $|\operatorname{Tr} M|=2$, but $M \neq \pm 1$,

$$
H=\left\{\left(\begin{array}{cc} 
\pm 1 & 0 \\
t & \pm 1
\end{array}\right) \mid t \in \mathbb{R}\right\}
$$

representatives:

$$
M= \pm\left(\begin{array}{ll}
1 & 0 \\
q & 1
\end{array}\right), q= \pm 1
$$

- Exceptional classes, $H=S L(2, \mathbb{R})$, representatives: $M= \pm 1$, these can be obtained as the $\omega \longrightarrow 0$ and $\omega \longrightarrow \frac{1}{2}$ limiting cases of the elliptic cases but the isotropy subgroups are larger.

According to section 1 for a given $M$ one has to find a $g$ then the corresponding $J$ current has to be studied whether it can be transformed to the gauge fixed form or not. The most straightforward case is when $M$ possesses a logarithm in $\operatorname{sl}(2, \mathbb{R})$. When this occurs the simplest choice is $g(x)=M^{\frac{x}{2 \pi}}$ to which corresponds the constant current $J_{0}=\frac{1}{2 \pi} \log M$. From the above listed representatives the elliptic monodromies have logarithms and from the others in the "+" case $M$, in the "-" case $-M$ has a logarithm and in the latter case a possible choice for $g$ is $g(x)=T_{\frac{1}{2}}(x)(-M)^{\frac{x}{2 \pi}}$ to which corresponds the non-constant current

$$
J_{\frac{1}{2}}=T_{\frac{1}{2}} J_{0} T_{-\frac{1}{2}}+T_{\frac{1}{2}}^{\prime} T_{-\frac{1}{2}}
$$

Remember that $T_{\frac{1}{2}}(x)$ is the matrix of rotation by $\frac{x}{2}$.

From $J_{0}$ and $J_{\frac{1}{2}}$ we will get a representative on each Kac-Moody component by applying $T_{n}$ to $J_{0}$ and (except for the elliptic case) to $J_{\frac{1}{2}}$. To simplify notation from now on let $n$ be a half-integer and

$$
J_{0}=\left(\begin{array}{cc}
j_{1} & j_{2}-j_{3} \\
j_{2}+j_{3} & -j_{1}
\end{array}\right)
$$

the constant element. We obtain a representative on each component of the Kac-Moody orbits by setting

$$
\begin{gathered}
J_{n}=T_{n} J_{0} T_{-n}+T_{n}^{\prime} T_{-n}= \\
=\left(n+j_{3}\right)\left(\begin{array}{cc}
0 & -1 \\
1 & 0
\end{array}\right)+j_{1}\left(\begin{array}{cc}
\cos (2 n x) & \sin (2 n x) \\
\sin (2 n x) & -\cos (2 n x)
\end{array}\right)+j_{2}\left(\begin{array}{cc}
-\sin (2 n x) & \cos (2 n x) \\
\cos (2 n x) & \sin (2 n x)
\end{array}\right)
\end{gathered}
$$

which is periodic also for half-integer $n$ as it should be.

To summarize, the $J_{n}$ in equation (13) for integer $n$ is a representative of the $n^{\text {th }}$ component of the Kac-Moody orbit corresponding to $M=e^{2 \pi J_{0}}$ monodromy, and for half-integer $n$ it is a representative of the $\left(n-\frac{1}{2}\right)^{t h}$ component of the Kac-Moody orbit corresponding to $M=-e^{2 \pi J_{0}}$ monodromy. As a reference let $J_{0}$ be on the $0^{\text {th }}$ component. 
To get the Virasoro representatives $J_{n}$ has to be transformed to the gauge fixed form with an $h \in$ $L S L(2, \mathbb{R})$ with zero winding number. Obviously the winding number of

$$
h=\left(\begin{array}{cc}
R^{-1} & \left(1+\frac{1}{n} R^{-2}\right) R^{\prime} \\
0 & R
\end{array}\right): S^{1} \longrightarrow S L(2, \mathbb{R})
$$

is zero and is easily checked to bring $J_{n}$ to the desired form as it is in the form of (10) (and has no singularity when $n=0$ ), and where

$$
R(x)=\frac{1}{\sqrt{n+j_{3}+j_{1} \sin (2 n x)+j_{2} \cos (2 n x)}} .
$$

This is only true of course if

$$
n+j_{3}+j_{1} \sin (2 n x)+j_{2} \cos (2 n x)>0,
$$

which has to be checked in each case. If this inequality holds the Virasoro representative turns out to be after straightforward calculations

$$
L=C+2 n\left(n^{2}+C\right) R^{2}+3 n^{2}\left(C-n^{2}-2 n j_{3}\right) R^{4},
$$

where $C=\frac{1}{2} \operatorname{Tr}\left(J_{0}\right)^{2}=j_{1}^{2}+j_{2}^{2}-j_{3}^{2}$. This is a surprisingly dense form if we take into account that it contains every possible case and besides it looks very similar to the representatives given in [17] obtained by very different methods. If the (14) inequality does not hold then conjugating $J_{0}$ by a constant element might change it in such a way that with the new $j_{1}, j_{2}$ and $j_{3}$ the inequality holds. This corresponds to choosing a different representative in the conjugacy class of the monodromy. As we will see this will occur in the hyperbolic case. In those cases when this can not be achieved it is quite easy to show through the analysis of the $\operatorname{det} h=1$ equation, where $h$ is of the $(10)$ form, that there is no transformation that brings $J_{n}$ to the gauge fixed form which means that the corresponding Kac-Moody component has no intersection with the constraint hypersurface.

For the sake of completeness we will give the vectorfields for each representative of the Virasoro orbit which leave it fixed under the coadjoint Virasoro action. The key observation was noted in section 1 and is that the $(2,1)$ component of a gauge preserving Kac-Moody transformation generates the conformal transformations, which means that for determining the isotropy vectorfields one has to look for the $(2,1)$ component of those infinitezimal Kac-Moody transformations which leave a particular gauge fixed current invariant. A general Virasoro representative was obtained from a constant $J_{0}$ by applying first $T_{n}$ and then $h$ to bring it to the gauge fixed form, so those elements which leave them invariant are obviously of the form $h T_{n} A T_{n}^{-1} h^{-1} \in L S L(2, \mathbb{R})$, where $A \in S L(2, \mathbb{R})$ commutes with the monodromy corresponding to $J_{0}$. Hence, the isotropy vectorfields are given by the $(2,1)$ component of $h T_{n} Y T_{n}^{-1} h^{-1} \in \operatorname{Lsl}(2, \mathbb{R})$, where $Y=\left(\begin{array}{cc}y_{1} & y_{2}-y_{3} \\ y_{2}+y_{3} & -y_{1}\end{array}\right)$ is any element of the Lie algebra of the commutant subgroup of $M$. The $(2,1)$ component turns out to be

$$
V=\frac{y_{3}+y_{2} \cos (2 n x)+y_{1} \sin (2 n x)}{n+j_{3}+j_{2} \cos (2 n x)+j_{1} \sin (2 n x)},
$$

which can be directly related to the results of [20]. Note that in any case when $L$ is non-constant and periodic with period $\frac{\pi}{n}$ a discrete $\mathbb{Z}_{n}$ subgroup must appear in the isotropy subgroup corresponding to rigid discrete rotations of the circle.

The case-by-case analysis proceeds as follows:

- Elliptic orbits, $n$ is an integer here and $R(x)=\frac{1}{\sqrt{n+\omega}}$ where $\omega \in(0,1)$ and $\omega \neq \frac{1}{2}$ so $n \geq 0$. The Virasoro representatives and stabilizing vectorfield (up to an irrelevant normalization factor) are

$$
L=-(n+\omega)^{2}, \quad V=1 .
$$

The $V$ vectorfield generates an $S^{1}$.

- Hyperbolic orbits, $n$ is a half-integer here and $R(x)=\frac{1}{\sqrt{n+b \sin (2 n x)}}$ where $b>0$. The expression under the square root can be negative for large $b$ but if $J_{0}$ is conjugated by $\left(\begin{array}{ll}1 & 0 \\ b & 1\end{array}\right)$ then from 
the new $J_{n}$ one obtains $R(x)=\frac{1}{\sqrt{n+b^{2}+b^{2} \cos (2 n x)+b \sin (2 n x)}}$ which is well defined, where $n \geq 0$. The Virasoro representatives and stabilizing vectorfield are

$$
L=b^{2}+\frac{2 n\left(n^{2}+b^{2}\right)}{n+b^{2}+b^{2} \cos (2 n x)+b \sin (2 n x)}+\frac{3 n^{2}\left(b^{2}-2 b^{2} n-n^{2}\right)}{\left(n+b^{2}+b^{2} \cos (2 n x)+b \sin (2 n x)\right)^{2}},
$$

and

$$
V=\frac{b+\sin (2 n x)+b \cos (2 n x)}{n+b^{2}+b \sin (2 n x)+b^{2} \cos (2 n x)},
$$

which for $n=0$ generates an $S^{1}$ and for $n \neq 0$ a one-parameter subgroup isomorphic to $\mathbb{R}$.

- Parabolic orbits, $n$ is a half-integer here and $R(x)=\frac{1}{\sqrt{n+\frac{q}{2 \pi} \cos ^{2}(n x)}}$. If $n=0$ only $q=1$ is allowed, otherwise $n>0$ and $q= \pm 1$ is arbitrary. The Virasoro representatives and stabilizing vectorfields are

$$
L=n^{3}\left(\frac{2}{n+\frac{q}{4 \pi}(1+\cos (2 n x))}-\frac{3\left(n+\frac{q}{2 \pi}\right)}{\left(n+\frac{q}{4 \pi}(1+\cos (2 n x))\right)^{2}}\right), V=\frac{1+\cos (2 n x)}{n+\frac{q}{4 \pi}(1+\cos (2 n x))},
$$

which again generates an $S^{1}$ for $n=0$ and a subgroup isomorphic to $\mathbb{R}$ for $n \neq 0$. The above form of $L$ explicitly shows that if $n=0$ then only one of the $q$ s are allowed because if it were not so then two distinct monodromies would correspond to the same $L=0$.

- Exceptional orbits, $n>0$ and is a half-integer here and $L$ can be obtained from the elliptic case as the $\omega \longrightarrow 0$ limit, but the stabilizing subgroup is larger. The Virasoro representatives and stabilizing vectorfields are

$$
L=-n^{2}, \quad V=1, \sin (2 n x), \cos (2 n x) .
$$

These $V$ s generate a $2 n$-fold "embedding" of $S L(2, \mathbb{R})$ into $\operatorname{Diff}\left(S^{1}\right)$.

It was noted in section 4 that the energy can have critical points only on those orbits which contain a constant representative. To study the stability of the critical points let us spell out the (6) second variation allowing now only such $K$ s that preserve the gauge. Having a constant $L$ at hand the form of these on the Lie algebra level are

$$
K=\frac{1}{2}\left(\begin{array}{cc}
\varepsilon^{\prime} & \varepsilon L-\varepsilon^{\prime \prime} \\
2 \varepsilon & -\varepsilon^{\prime}
\end{array}\right)
$$

according to the infinitezimal version of formula $(10)$ where $\varepsilon: S^{1} \longrightarrow \mathbb{R}$ is some function. Calculation of the diagonal element of the (6) second variation gives

$$
\delta \delta E=2 \int_{0}^{2 \pi}\left(L \varepsilon^{\prime 2}+\frac{1}{4} \varepsilon^{\prime \prime 2}\right) \frac{d x}{2 \pi}
$$

which is easily seen to be non-negative for any $\varepsilon$ if and only if $L \geq-\frac{1}{4}$.

\section{$6 S L(3, \mathbb{R})$ Kac-Moody and Zamolodchikov algebra}

In the $S L(3, \mathbb{R})$ WZNW model two fields survive the reduction, the $W_{2}=L$ energy-momentum tensor and the $W_{3}=W$ field. There are two free functions in the infinitezimal version of the (10) gauge preserving transformations out of which the second component of $\mathbf{a}_{1}$ generates the conformal and the first component the $W$-transformations. Applying such an infinitezimal transformation to a gauge fixed current one can read off the Poisson brackets [1]:

$$
\begin{aligned}
\{L(x), L(y)\}= & L^{\prime}(x) \delta(x-y)-2 L(x) \delta^{\prime}(x-y)-2 \delta^{\prime \prime \prime}(x-y) \\
\{L(x), W(y)\}= & W^{\prime}(x) \delta(x-y)+3 W(x) \delta^{\prime}(x-y)- \\
& -(L(x) \delta(x-y))^{\prime \prime}+\delta^{\prime \prime \prime \prime}(x-y) \\
\{W(x), W(y)\}= & \left(\frac{2}{3} L^{\prime}(x) L(x)+W^{\prime \prime}(x)-\frac{2}{3} L^{\prime \prime \prime}\right) \delta(x-y)+ \\
& +\left(\frac{2}{3} L^{2}(x)+2 W^{\prime}(x)-2 L^{\prime \prime}(x)\right) \delta^{\prime}(x-y)- \\
& -2 L^{\prime}(x) \delta^{\prime \prime}(x-y)-\frac{4}{3} L(x) \delta^{\prime \prime \prime}(x-y)+\frac{2}{3} \delta^{\prime \prime \prime \prime \prime}(x-y)
\end{aligned}
$$


where the $W$ field is not primary but if gauge fixing is changed from the Wronskian to the highest weight one [1, 2], which is essentialy a shift, $\widetilde{W}=W-\frac{1}{2} L^{\prime}$, then the Poisson brackets for the new fields show that $\widetilde{W}$ is a primary field with conformal weight 3 and that its bracket with itself gives polinomial terms in the energy-momentum tensor and happens to be identical to the Zamolodchikov algebra.

As the fundamental group of $S L(3, \mathbb{R})$ is $\mathbb{Z}_{2}$ each Kac-Moody orbit consists of two connected components. The

$$
T_{n}(x)=\left(\begin{array}{ccc}
\cos (n x) & -\sin (n x) & 0 \\
\sin (n x) & \cos (n x) & 0 \\
0 & 0 & 1
\end{array}\right)
$$

transformation determines the non-trivial element in $\pi_{1}(S L(3, \mathbb{R}))$ if $n$ is odd. This means that for $n$ odd $T_{n}$ shifts between the two components, for $n$ even $T_{n}$ preserves them. This will result the interesting consequence that on some Kac-Moody orbits and on the corresponding $W$-leaves there will be infinitely many critical points of the energy but at most one will be a minimum.

The exploration of the $W$-leaves will proceed in a completely analogous way as in the previous section. Since the fundamental group has two elements there corresponds at least two symplectic leaves to each monodromy matrix but it is known from the context of homotopy types of non-degenerate curves on projective space or sphere that in some cases this classification is not complete as sometimes the constraint intersects one of the two components of the Kac-Moody orbits in two pieces, which will mean that to such a monodromy 3 leaves correspond $[6,9]$.

A complete list of conjugacy classes with the isotropy subgroups follows. Note that the isotropy subgroups have dimension 2,4 or 8 so an $(L, W)$ pair can be left fixed by only 2,4 or 8 linearly independent $W$-transformations.

- Hyperbolic classes, the diagonalizable matrices with 3 distinct, real eigenvalues belong here.

$$
H=\left\{\left(\begin{array}{ccc}
t & 0 & 0 \\
0 & r & 0 \\
0 & 0 & (t r)^{-1}
\end{array}\right) \mid t, r \neq 0\right\} \simeq \mathbb{R}^{*} \times \mathbb{R}^{*}
$$

representatives:

$$
M=\left(\begin{array}{ccc} 
\pm e^{2 \pi a} & 0 & 0 \\
0 & \pm e^{2 \pi b} & 0 \\
0 & 0 & e^{-2 \pi(a+b)}
\end{array}\right) .
$$

- Degenerate hyperbolic classes, the diagonalizable matrices with 2 distinct, real eigenvalues belong here.

$$
H=\left\{\left(\begin{array}{ccc}
t & r & 0 \\
u & v & 0 \\
0 & 0 & (t v-r u)^{-1}
\end{array}\right) \mid t v-r u \neq 0\right\} \simeq G L(2, \mathbb{R}),
$$

representatives:

$$
M=\left(\begin{array}{ccc} 
\pm e^{2 \pi b} & 0 & 0 \\
0 & \pm e^{2 \pi b} & 0 \\
0 & 0 & e^{-4 \pi b}
\end{array}\right), b \neq 0 \text { in the "+" case } .
$$

- Elliptic classes, those matrices belong here which are diagonalizable over the complex numbers with one real and a complex and its conjugate eigenvalue.

$$
H=\left\{\left(\begin{array}{ccc}
t & -r & 0 \\
r & t & 0 \\
0 & 0 & \left(t^{2}+r^{2}\right)^{-1}
\end{array}\right) \mid t^{2}+r^{2} \neq 0\right\} \simeq \mathbb{C}^{*},
$$

representatives:

$$
M=\left(\begin{array}{ccc}
e^{2 \pi b} \cos (2 \pi \omega) & -e^{2 \pi b} \sin (2 \pi \omega) & 0 \\
e^{2 \pi b} \sin (2 \pi \omega) & e^{2 \pi b} \cos (2 \pi \omega) & 0 \\
0 & 0 & e^{-4 \pi b}
\end{array}\right), \omega \in(0,1), \omega \neq \frac{1}{2} .
$$


- 1. parabolic classes, matrices with one degenerate Jordan block belong here.

$$
H=\left\{\left(\begin{array}{ccc}
t & r & 0 \\
0 & t & 0 \\
0 & 0 & t^{-2}
\end{array}\right) \mid t \neq 0\right\} \simeq \mathbb{R} \times \mathbb{R}^{*}
$$

representatives:

$$
M=\left(\begin{array}{ccc} 
\pm e^{2 \pi b} & 1 & 0 \\
0 & \pm e^{2 \pi b} & 0 \\
0 & 0 & e^{-4 \pi b}
\end{array}\right), b \neq 0 \text { in the "+" case }
$$

- 2. parabolic class.

$$
H=\left\{\left(\begin{array}{ccc}
t & r & u \\
0 & t & 0 \\
0 & v & t^{-2}
\end{array}\right) \mid t \neq 0\right\} \simeq \mathbb{R}^{3} \times \mathbb{R}^{*}
$$

representative:

$$
M=\left(\begin{array}{lll}
1 & 1 & 0 \\
0 & 1 & 0 \\
0 & 0 & 1
\end{array}\right)
$$

- 3. parabolic class.

$$
H=\left\{\left(\begin{array}{ccc}
1 & t & r \\
0 & 1 & t \\
0 & 0 & 1
\end{array}\right) \mid t, r \in \mathbb{R}\right\} \simeq \mathbb{R}^{2}
$$

representative:

$$
M=\left(\begin{array}{lll}
1 & 1 & 0 \\
0 & 1 & 1 \\
0 & 0 & 1
\end{array}\right)
$$

- Exceptional class.

$$
H=S L(3, \mathbb{R}) \text {, representative: } M=1 \text {. }
$$

At the hyperbolic, degenerate hyperbolic and the 1. parabolic class the signs are either both positive or both negative.

The classification of $W$-leaves by the fundamental group is complete in the "-" version of the hyperbolic, the degenerate hyperbolic, and the 2. parabolic case. In the others the constraint intersects one of the two Kac-Moody components in two piece, more concretely in the exceptional case $n=1$ corresponds to a separate leaf, while all other odd $n$ correspond to a second, all even $n$ to a third leaf. In the remaining cases $n=0$ constitutes to a separate leaf, all other even $n$ to a second and all odd $n$ to a third leaf [6, 9].

The given representatives possess logarithms except for the 1. parabolic and the "-" version of the hyperbolic classes. The latter case was analyzed in $[2]$ and the authors did not find constant $L$ and $W$ representatives for the corresponding leaf which is not surprising as we have noted in section 3 that if a monodromy does not have a logarithm then the corresponding orbit can not have a constant representative.

In the "-" cases the trick used for $S L(2, \mathbb{R})$ will be adopted in the upper left block, i.e. take the opposite sign of the upper left $2 \times 2$ block of $M$, call it $M_{-}$which possess a logarithm and then the monodromy of $g(x)=T_{\frac{1}{2}}(x) M_{-}^{\frac{x}{2 \pi}}$ will be $M$ where $T_{\frac{1}{2}}(x)$ is the matrix of rotation by $\frac{x}{2}$ in the upper left block. On the resulting matrices the $T_{n}$ matrix must be applied but note again that for $n$ even we will stay in the original component. The resulting matrices should be transformed into the gauge fixed form by a Kac-Moody transformation that determines the trivial element of $\mathbb{Z}_{2}$.

Let us analyze the stability of the critical points on the $W$-leaves which is important for determining the classical h. w. states. Substituting the gauge preserving infinitezimal transformations into the (6) diagonal element of the second variation of the energy gives:

$$
\delta \delta E=\int_{0}^{2 \pi}\left(\begin{array}{ll}
\varepsilon_{1}^{\prime} & \varepsilon_{2}^{\prime}
\end{array}\right)\left(\begin{array}{cc}
\frac{2}{3}\left(L-\frac{d^{2}}{d x^{2}}\right)^{2} & 3 W-\left(L-\frac{d^{2}}{d x^{2}}\right) \frac{d}{d x} \\
3 W+\left(L-\frac{d^{2}}{d x^{2}}\right) \frac{d}{d x} & 2\left(L-\frac{d^{2}}{d x^{2}}\right)
\end{array}\right)\left(\begin{array}{l}
\varepsilon_{1}^{\prime} \\
\varepsilon_{2}^{\prime}
\end{array}\right) \frac{d x}{2 \pi}
$$

supposed $L$ and $W$ are constant, where $\varepsilon_{1}$ and $\varepsilon_{2}$ are the functions generating the conformal and $W$ transformations. This quadratic form is non-negative for any $\varepsilon_{1}$ and $\varepsilon_{2}$ if and only if

$$
(L+1)^{2}(4 L+1) \geq 27 W^{2}
$$


holds for the constant representatives, which shows that $L \geq-\frac{1}{4}$ is necessary just as in the pure Virasoro case.

The straightforward calculation gives in the individual cases the following, where we have not bothered writing out explicitly the non-constant representatives as they are quite complicated and have no transparent physical meaning.

- Hyperbolic orbits. In the $n=0$ and "+" case one has constant representatives which can be obtained by constant Kac-Moody transformations:

$$
L=a^{2}+a b+b^{2}, \quad W=-a b(a+b)
$$

which are all minima of the energy. If $n \neq 0$ there are only non-constant $L$ and $W$ representatives.

- Degenerate hyperbolic orbits. In this case $n$ is a half-integer, the classification is complete by the fundamental group and we have constant representatives for the $W$-leaves:

$$
L=3 b^{2}-n^{2}, \quad W=-2 b\left(b^{2}+n^{2}\right) .
$$

Note again that these elements describe 4 different leaves, according to whether $n \neq 0$ is an integer or half-integer (the monodromies are different) and whether $n$ is even or odd in case it is an integer, or $n-\frac{1}{2}$ is even or odd in case $n$ is a half-integer (same monodromy but different Kac-Moody component). Now any leaf possesses infinitely many critical points but only in the "-" case will one find a minimum and it only happens if $n=\frac{1}{2}$ and $b$ arbitrary and the minimum is degenerate. Note that these classical h. w. states are $G L(2, \mathbb{R})$ invariant so they are good candidates for a true vacuum in CFT.

- Elliptic orbits. Here $n$ is an integer and one obtains constant representatives for any $W$-leaf:

$$
L=3 b^{2}-(\omega+n)^{2}, \quad W=-2 b\left(b^{2}+(\omega+n)^{2}\right), \quad \omega \in(0,1), \omega \neq \frac{1}{2} .
$$

In these cases the classification by the fundamental group is not complete, the constraint intersects the component corresponding to even $n$ in two piece, but the $W$-leaf corresponding to odd $n$ is connected and the (16) representatives determine the same $W$-leaf for all odd $n$ which means that on these leaves the energy has infinitely many critical points. The critical points are minima only if $n=0$ and $|\omega|<\frac{1}{2}$ and $b$ arbitrary.

- 1. parabolic orbits. These have constant representatives for $n=0$ and they are given by

$$
L=3 b^{2}, \quad W=-2 b^{3},
$$

which are minima of the energy for arbitrary $b$.

- 2. parabolic orbits. None of these orbits have constant representatives.

- 3. parabolic orbit. The constant representative is

$$
L=0, \quad W=0,
$$

which is a minimum of the energy.

- Exceptional orbit. The leaf corresponding to this orbit has infinitely many constant representatives,

$$
L=-n^{2}, \quad W=0,
$$

where $n \neq 0$ is an integer, but none of these are minima of the energy.

Note again that a constant representative with non-negative second variation of the energy is a classical h. w. state. One hopes to construct a consistent highest weight representation space on these leaves on which the quantized version of the $W$-algebra will act in CFT. The uniqueness of the vacuum in CFT is an important issue and we have established that if there is a critical point of the energy on a leaf then at most one will give rise to a vacuum as at most one of the possibly many critical points can be a stable minimum. Those leaves which do not contain critical points, that means on which one can not find a constant representative, are physically less important as one can not associate a heighest weight type representation of the $W$-algebra to these. 


\section{Conclusions and outlook}

We have analyzed in this paper the classical geometry of $W$-algebras in the context of reduced $S L(n, \mathbb{R})$ WZNW models. We split the WZNW field as

$$
g(x, t)=g_{+}\left(x_{+}\right) g_{-}\left(x_{-}\right)
$$

and concentrated on one chiral half of the original WZNW theory only. As a consequence of the splitting the chiral theory inherited a global $G$-symmetry $g_{+} \rightarrow g_{+} h, h \in G$. Both the unconstrained and constrained currents are invariant under this global symmetry, since

$$
J_{+}=\left(\partial_{+} g\right) g^{-1},
$$

that is the fields $W_{2}, \ldots, W_{n}$ are $S L(n, \mathbb{R})$ invariant. We fixed this gauge symmetry, however, by demanding $g(0)=e$ and establishing a one to one correspondence between the current $J_{+}$and the group valued field $g_{+}$. In the reduced case this means that the fields $W_{2}, \ldots, W_{n}$ can be determined from the last row of $g$, that is from a non-degenerate curve in $\mathbb{R}^{n}$ as we have seen. If we had not fixed the gauge we would have the global $S L(n, \mathbb{R})$ gauge symmetry. The geometry of this symmetry was discussed in detail in [29]. Let's summarize their results. This symmetry acts naturally on the space of non-degenerate curves and the generators of the classical $\mathrm{W}$-algebra, that is the components of the constrained currents $W_{2}, \ldots, W_{n}$ are invariant. Since they can be built up from the non-degenerate curve as differential polynomials which are clearly invariant with respect to the gauge symmetry they can be interpreted as "affine" invariants. It was also shown that they are the only invariants and determine the curve modulo gauge transformations. The Virasoro generator is a second order invariant which is called "affine" curvature, the next $W$-generator can be interpreted as "affine" torsion, etc.

Here we have concentrated on the classification of $W$-leaves and the issue of classical h. w. states in one chiral half. We can extend our results for the other chiral half of the theory since analogous formulae hold for them. Putting the two chiral halfs together the solutions of the reduced WZNW models, namely the Toda models, can be obtained. Our classification of the leaves leads to a classfication of the $W$ inequivalent Toda solutions as it was described in detail in [17] for the global Liouville equation. Our method in principle is able to give explicit representatives on each leaf which fact was put in practice in the search for classical h. w. type representations. All these considerations were carried out for $n=2$ and $n=3$ which corresponds to the Virasoro and Zamolodchikov algebra respectively. We have found that there is at most one classical h. w. state on any $W$-leaf and in the Zamolodchikov algebra case also determined the $S L(2, \mathbb{R})(G L(2, \mathbb{R})$ in fact) invariant highest weight states which are the best candidates for a true vacuum in CFT.

There is a very interesting and delicate question, how the classical representions are related to the quantum highest weight representations. One possible approach is to take the classical equations of motion and try to quantize them. This idea was used in [2, 3, 胞 and they found that consistent quantization results in $W$-minimal models, where the classical Toda field corresponds to the simplest nontrivial completely degenerate representation of the quantum $W$-algebra. Later it was extended by taking higher dimensional representions of the reduced WZNW model to obtain other representions in the quantum case in $\| \mathbb{8} \mid$. It is not clear however how this representions are related to the symplectic leaves of the classical $W$-algebra even in the simplest Virasoro case, where only a conjecture from Witten gives some hint [18]. A proper description would be the geometric quantization of the leaves classified in this paper. This is a difficult problem and is unsolved even in the finite $W$-algebra case except for the simplest situation [|]|.

\section{Acknowledgements}

The work of Z. B. was supported by the grants OTKA D25517 and T029802.

\section{References}

[1] J. Balog, L. Fehér, L. O'Raifeartaigh, P. Forgács, A. Wipf: Toda Theory and $W$-algebra from the Gauged WZNW Point of View, Ann. Phys. (NY) 203 (1990) 76

[2] Z. Bajnok, L. Palla, G. Takács: $A_{2}$ Toda theory in reduced WZNW framework and the representations of the $W$-algebra, Nucl. Phys. B385 (1992) 329-360

[3] J. Balog, L. Palla: Locality Problem for the Liouville Field, Phys. Lett. B274 (1992) 323-330 
[4] L. Fehér, L. O'Raifeartaigh, P. Ruelle, I. Tsutsui: On Hamiltonian Reductions of the Wess-ZuminoNovikov-Witten Theories, Phys. Rep. 222, No. 1 (1992) 1-64. North-Holland

[5] Z. Bajnok: $C_{2}$ Toda theory in the reduced WZNW framework, Int. J. Mod. Phys. A9 (1994) 5387-5408

[6] V. Yu. Ovsienko, B. A. Khesin: Symplectic Leaves of the Gel'fand-Dikii Brackets and Homotopy Classes of Nondegenerate Curves, Funct. Anal. Appl. 24(1), (1990) 33

[7] Z. Bajnok, D. Nógrádi, D. Varga, F. Wágner: Geometric quantisation of the global Liouville mechanics, J. Phys. A32, (1999) 7477-7481

[8] Z. Bajnok: A new approach to the correlation functions of $W$-algebras, hep-th/9906185

[9] B. A. Khesin, B. Z. Shapiro: Homotopy classification of nondegenerate quasiperiodic curves on 2sphere, Publications de l'Institut Mathematique, nouvelle serie, 66(80), 1999, 127-156.

[10] J. A. Little: Nondegenerate homotopies of curves on the unit 2-sphere, J. Diff. Geom. 4 (1970) 339-348

[11] I. M. Gel'fand, L. A. Dikii, Funk. Anal. Priloz. 10 (1976) 13.

[12] I. M. Gel'fand, L. A. Dikii: The Family of the Hamiltonian Structures that are Connected with Integrable Nonlinear Equations, Inst. Prikl. Matematiki, No. 136 (1978)

[13] P. Bouwknegt, K. Schoutens: $W$-symmetry in conformal field theory, Phys. Rep. 223 (1993) 183.

[14] V. A. Fateev, S. L. Lukyanov: Additional symmetries and exactly soluble models in two-dimensional conformal field theory, Sov. Sci. Rev. A. Phys. Vol. 15 (1990) 1-117.

[15] S. L. Luk'yanov: Quantization of the Gel'fand-Dikii bracket, Funkts. Anal. Prilozhen. 22 No. 4. (1988) $1-10$

[16] E. Frenkel, V. G. Kac, M. Wakimoto: Characters and fusion rules for $W$-algebras via quantized Drinfeld-Sokolov reductions, Comm. Math. Phys. 147 (1992) 295,

[17] J. Balog, L. Fehér, L. Palla: Coadjoint Orbits of the Virasoro Algebra and the Global Liouville Equation, Int. Jour. Mod. Phys. A Vol. 13 No.2 (1998) 315-362

[18] E. Witten: Coadjoint Orbits of the Virasoro Group, Commun. Math. Phys. 114, (1988) 1-53

[19] G. Segal: Unitary Representation of some Infinite Dimensional Groups, Commun. Math. Phys. 80 (1981) 301-342

[20] A. Gorskii, B. Roy, K. Selivanov: Large gauge transformations and special orbits of the Virasoro group, Pis'ma Zh, Eksp. Teor. Fiz. 53 No. 1 (1991) 59-62

[21] J. de Boer, F. Harmsze, T. Tjin: Non-linear finite $W$-symmetries and applications in elementary systems, Phy. Rep. 272 (1996) 139-214

[22] T. Tjin: Finite $W$-algebras, Phys. Lett. B292 (1992) 60

[23] L. Fehér, I. Tsutsui: Regularization of Toda lattices by Hamiltonian reduction, Jour. Geom. Phys. 21 (1997) 97-135

[24] P. Goddard, D. Olive: Kac-Moody and Virasoro algebras in relation to quantum physics, Int. Jour. Mod. Phys. A. Vol. 1. No. 2 (1986) 303-414

[25] A. B. Zamolodchikov: Additional symmetries in the two-dimensional quantum conformal theory of fields, Teor. Mat. Fiz. 65 No. 3 (1985) 347-359

[26] V. A. Fateev, A. B. Zamolodchikov: Conformal quantum field theory models in two dimensions having $Z_{3}$ symmetry, Nucl. Phys. B280 (1987) 644-660

[27] J. L. Gervais, Y. Matsuo: W-geometries, Phys. Lett. B274 (1992) 309

[28] J. L. Gervais, Y. Matsuo: Classical $A_{n} W$-geometry, Comm .Math. Phys. 152 (1993) 317-368

[29] G. Sotkov, M. Stanishkov: Affine geometry and $W_{n}$-gravities, Nucl. Phys. B356 (1991) 439-468 
[30] A. Bilal, V. V. Fock, I. I. Kogan: On the origin of $W$-algebras, Nucl. Phys. B359 (1991) 635-672

[31] K. Gawedzki: Conformal field theory: a case study, hep-th/9904145

[32] M. B. Halpern, E. Kiritsis, N. A. Obers, K. Clubok: Irrational Conformal Field Theory, Phys. Rep. 265 (1996) 1-138

[33] A. Pressley, G. Segal: Loop Groups, Clarendon Press Oxford 1986 